advance human health OBJECTIVES/GOALS: Mount Sinai Targeted Healthcare Innovation Fellowship (THRIVE) is a 9-month program for participants from diverse professional backgrounds to develop HealthTech innovations related to COVID-19. The program is designed to provide an experiential team science platform for fellows to take an idea from concept to commercially viable innovation. METHODS/STUDY POPULATION: Following a competitive application process, 16 THRIVE fellows comprise four teams working collaboratively in an online forum with input from experts in the field. Success of the program will be evaluated by: assessing pre- and post- collaborative research orientation among THRIVE fellows using the ROI scale1 using social network analysis (SNA) to investigate the social networks of THRIVE fellows to capture patterns of communication and collaboration related to innovation development exploring participant experiences of group formation, teamwork and collaboration related to innovation development using one-to-one semi-structured interview determining team success in innovation development, measured by number of publications, funding awarded, provisional patents and viable products. RESULTS/ANTICIPATED RESULTS: Paired t-tests will determine whether collaborative orientation of THRIVE fellows changes prevs. post- program participation, indicating changes in attitude toward multidisciplinary team work. SNA will be used to describe structural patterns of communication that occur at individual and group levels. Network-level indices will provide insight into patterns of communication that exist in innovation development: degree centrality (number of connections per individual), betweenness centrality (number of bridges to others in a network), closeness centrality (closeness to others in a network). We will also test for associations between network characteristics and team success. DISCUSSION/ SIGNIFICANCE OF FINDINGS: Understanding patterns of formal and informal relationships, interactions, and perceptions of the collaborative process among individuals in THRIVE teams will elucidate whether such a program can provide an effective forum for team science and innovation development related to COVID-19.

16506

Recognizing Interdisciplinary Collaborative Research in Promotion and Tenure Processes

Brenda K. Zierler ${ }^{1}$, Nicole Summerside ${ }^{2}$, Jennifer Sprecher ${ }^{3}$, Erin Blakeney $^{3}$, Mia Vogel ${ }^{3}$, Frances $\mathrm{Chu}^{4}$ and Jonathan D. Posner ${ }^{5}$ ${ }^{1}$ Department of Biobehavioral Nursing and Health Informatics, Center for Health Sciences Interprofessional Education, Research, and Practice, University of Washington; ${ }^{2}$ Department of Biobehavioral Nursing and Health Informatics, Center for Health Sciences Interprofessional Education, University of Washington; ${ }^{3}$ Department of Biobehavioral Nursing and Health Informatics, Center for Health Sciences Interprofessional Education University of Washington; ${ }^{4}$ Department of Mechanical Engineering, Department of Chemical Engineering, Department of Family Medicine, University of Washington

ABSTRACT IMPACT: Recognizing Interdisciplinary Collaborative Research in Promotion and Tenure Processes OBJECTIVES/ GOALS: Academic institutions have traditionally focused on individual achievements for promotion. We present our effort on identifying and measuring attitudes on promotion and tenure (PT) criteria that values and rewards interdisciplinary research (IR). We have developed a toolkit to facilitate the recognition of IR in PT processes. METHODS/STUDY POPULATION: Our group reviewed appointment, promotion and tenure (APT) policies from each of the six Health Science Schools and the College of Engineering at the University of Washington (UW) to assess language of objective criteria and attributes of IR to guide APT committees in the evaluation of interdisciplinary researchers. We surveyed faculty about their attitudes relating to IR within the context of promotion and tenure. Interviews of department chairs and administrators about institutional policies and infrastructure that supports or inhibits IR, and current best practices, were conducted. We have developed toolkits for junior faculty, department chairs, external reviewers, and APT committees to facilitate rewarding IR at promotion. RESULTS/ANTICIPATED RESULTS: Review of APT policies found criteria that recognizes IR for APT in three schools. 118 faculty responded to the survey (44\% Professor, 26\% Associate, and 37\% eligible for APT committees). The majority of faculty reported they were currently conducting IR (95\%), considered IR important (98\%), and believed the UW faculty code should encourage IR $(85 \%)$. Although a vast majority considered their units supportive of IR (>80\%), less than half (43\%) reported that their APT criteria provided examples that included participation in IR. Our survey also found that APT committees were challenged about best practices to reward IR, APT external reviewers struggle to evaluate individual vs team contributions, and individual faculty are challenged to describe contributions for APT within context of an interdisciplinary team. DISCUSSION/SIGNIFICANCE OF FINDINGS: IR is conducted and valued by UW faculty; however, current structures, policies, and APT code do not facilitate IR for promotion and tenure. We have developed a toolkit for promotion-eligible faculty, chairs, external reviewers, and APT committees to facilitate IR. Our goal is to modify UW faculty code and unit APT criteria to recognize and reward IR.

59182

\section{An exploratory analysis of network bridges in translational research; a case study of research grants collaboration networks at University of Rochester School of Medicine and Dentistry}

Reza Yousefi Nooraie, Elizabeth Wayman and Ann Dozier University of Rochester Medical Center

ABSTRACT IMPACT: This analysis helps disentangle various paths to translational collaboration, with implications for departmental capacity building and support. OBJECTIVES/GOALS: Studies that bridge research collaboration networks are cross-disciplinary and translational. We explored the characteristics of researchers and their collaboration patterns in bridging research grants at University of Rochester School of Medicine and Dentistry. METHODS/STUDY POPULATION: the database of sponsored research grants from 2011 to 2018, obtained from an internal University database was transformed into a two-mode network of grant-to-investigator. Grants at 90th percentile and above of normalized two-mode betweenness centrality were defined as 'bridging grants'. For each grant we extracted the gender, academic rank, academic degree, affiliating department, and centrality-status (being at 75th percentile of degree centrality in one-mode collaboration network) of the Principal Investigator (PI), as well as the number of co-investigators (CI) and the existence of central actor(s) in the research team. RESULTS/ANTICIPATED RESULTS: Out of 2491 sponsored grants, 250 were 'bridging grants'. The significant predictors of bridging were centrality of PI, existence of central CI(s), PI holding $\mathrm{PhD}$, and larger number of CIs. The PI's academic rank (being full professor) and gender were not significant predictors. Among bridging grants 79 included both central PI and CIs (central actors group) 
and 60 included no central actor on the team. In the latter group, more PIs were clinical faculty and fewer were full professors. Network analysis of affiliating departments showed that Medicine was the prominent actor in the central actors group, while the network of no-central actor group was more fragmented with Neurology as central. DISCUSSION/SIGNIFICANCE OF FINDINGS: Widely recognized researchers are more likely to collaborate with each other in bridging studies possibly marginalizing less experienced peers. Bridging grants led by less central researchers, often clinician-scientists, may thrive where supportive culture and departmental facilities exist.

\section{Health Equity \& Community Engagement}

\section{8}

\section{Bridging Gaps to Equalize Community-Academic Partnership: A Comparison of Capacities With Research Needs Across CTSA Program Hubs}

Bonnie Spring, David Moskowitz, Angela F. Pfammatter, Ruchi

Patel, Hannah Rumsey

Northwestern University Feinberg School of Medicine

ABSTRACT IMPACT: Our research identifies key opportunities for increased cross-CTSA collaboration, as a means to improve community-research cooperation and better CBPR practices. OBJECTIVES/ GOALS: Currently, team science training prioritizes developing the collaborative competencies of interdisciplinary scientists to work with each other and, more recently, with communities. Community-facing team science resources are scarce but present among some CTSAs, suggesting that capacity gaps might be remedied through cross-hub collaboration. METHODS/STUDY POPULATION: We reviewed online information provided by the 62 current CTSAs to identify: (1) which hubs engage in community research, and (2) what resources the hubs utilize to orient, train, and support community stakeholders as research partners. We then examined the capacities of the collectively available CTSA resources to address needed knowledge, skills, and attitudes that communityengaged researchers have identified as essential for communitybased stakeholders to partner equally in research. Finally, we explored practical challenges in team-based dynamics (e.g., interpersonal difficulties, expertise gaps, resource management) that may facilitate or hinder communities' research endeavors, and suggest resources that CTSAs might implement to facilitate team science dynamics. RESULTS/ANTICIPATED RESULTS: Hubs $(n=59)$ have community engagement programs, 12 of which provide communitybased participatory research toolkits. Toolkits vary from basic checklists to fully developed modules. Some hubs also offer consultation services and partner match-making. Learning objectives include: outcome definition, logic models, and goal-setting. Learning resources remain underdeveloped to help communities appreciate the benefits of research engagement and convince academic partners of the value of real-world knowledge and community improvement relative to scientific advancement. Also lacking is easily accessible support to understand the research process, build verifiable trust, maintain bidirectional knowledge and assets, and implement consistent, best practice methodological and reporting protocols. DISCUSSION/SIGNIFICANCE OF FINDINGS: Gaps between current hub offerings and community needs suggest prioritizing creation of resources whose learning objectives highlight the benefits of research engagement for community partners; foster mutual values affirmation between partners; and offer tools that build warranted community-researcher rapport.

Translational Science, Policy, \& Health Outcomes Science

46733

Strategy for Effective Team Formation: A Case Study of Rutgers' Big Ideas Initiative

Ziyad Razeq, Biju Parekkadan, Nancy Reichman and Edmund Lattime

Rutgers University

ABSTRACT IMPACT: This study will provide valuable insight regarding the effectiveness of a top-down approach for team formation. OBJECTIVES/GOALS: Rutgers' Big Ideas is a philanthropic initiative designed to gather team science ideas and present them to donors. We intend to evaluate this Team Science intervention and determine its feasibility in catalyzing the inception of team formation. We will explore the composition of teams that are formed using this particular method and team outcomes. METHODS/ STUDY POPULATION: Our group will first evaluate the themes that were covered by the initial 210 submissions as well as the 40 ideas chosen to be presented at the Big Ideas Symposium. We will also be taking a look at the donor population that these ideas were presented to. Then, we will evaluate the $8-12$ winning teams that were chosen to move forward. We will compare various success metrics of the 8-12 teams that were chosen compared to the 40 ideas that had not been chosen. RESULTS/ANTICIPATED RESULTS: Encouraging team science through an initiative such as the Big Ideas forum is not only feasible, but also highly effective in creating resilient teams that show prolonged productivity in fundraising, publications, and other academic metrics. DISCUSSION/ SIGNIFICANCE OF FINDINGS: Team Science is an exciting movement with immense potential. To that extent, this study seeks to discuss ways that academic leadership can inspire and foster effective team science collaboration. Concurrently, our case review lays the groundwork for further improvements to Team Science initiatives.

66361

\section{TL1 Team Approach to Predicting Response to Spinal Cord Stimulation for Chronic Low Back Pain* \\ Kyle See ${ }^{1}$, Rachel Ho${ }^{2}$, Stephen Coombes ${ }^{2}$ and Ruogu Fang ${ }^{1}$ \\ ${ }^{1}$ University of Florida Biomedical Engineering; ${ }^{2}$ University of Florida Applied Physiology and Kinesiology}

ABSTRACT IMPACT: Understanding how spinal cord stimulation works and who it works best for will improve clinical trial efficacy and prevent unnecessary surgeries. OBJECTIVES/GOALS: Spinal cord stimulation (SCS) is an intervention for chronic low back pain where standard interventions fail to provide relief. However, estimates suggest only $58 \%$ of patients achieve at least $50 \%$ reduction in their pain. There is no non-invasive method for predicting relief provided by SCS. We hypothesize neural activity in the brain can fill 